\title{
Management of lung cancer in South East Scotland
}

\author{
R J Fergusson, A Gregor, R Dodds, G Kerr, on behalf of the South East of Scotland \\ Lung Cancer Group and Lothian Audit Office
}

\begin{abstract}
Background - There are few reports which describe the management of unselected groups of patients with lung cancer. This study was undertaken to audit prospectively the presentation, diagnosis, management, and outcome of patients presenting with lung cancer in South East Scotland.

Methods - Data were recorded on all patients with newly diagnosed lung cancer who presented to a multidisciplinary group of clinicians over a 12 month period. Subsequent follow up data on treatment and survival were collected.

Results - Six hundred and twenty two patients were registered, $80 \%$ of whom were referred from primary care. There was a considerable variation in the length of history, but the diagnosis was rapidly made after referral $(87 \%$ within two weeks). In $82 \%$ of patients the pathological examination was positive; $70 \%$ were treated with palliative intent. Only $36 \%$ of patients who underwent surgery had computed tomographic scanning and 55\% had sampling of mediastinal nodes. A wide variety of regimens was used for treatment with radiotherapy and chemotherapy, and follow up data were difficult to obtain in these patients. Survival was poor in all patients treated with palliative intent.

Conclusions - This audit confirms the importance of previously noted prognostic factors. Significant variation in referral practice, diagnostic and management evaluation has been shown. The data serve as a useful background for the formation of local management guidelines.

(Thorax 1996;51:569-574)
\end{abstract}

Keywords: lung cancer, management, survival, surgery, radiotherapy, chemotherapy, palliation.

At a time when national guidelines for the management of common cancers are being formulated it is important to note how unselected patients are currently managed. Few population-based studies are available that provide detailed documentation of management processes and outcome for common cancers in general, and lung cancer in particular.

The South East of Scotland Lung Cancer Group (SESLCG) is a multidisciplinary group of respiratory physicians, thoracic surgeons, pathologists and radiation and medical oncologists responsible for the diagnosis and management of most of the cases of lung cancer in South East Scotland (Lothian, Fife and Borders regions, population base 1.1 million) referred for specialist respiratory or thoracic surgical opinion. The group had previous experience of collecting patient and tumour-related information at the time of diagnosis ${ }^{1}$ and decided to examine prospectively the presentation, diagnosis, management, and outcome of patients presenting to its individual members over a 12 month period.

\section{Methods}

All patients with newly diagnosed lung cancer presenting to chest physicians in the group were registered at the time of their diagnosis for a 12 month period commencing on 1 March 1991. Data recorded on case report sheets specifically designed for this study included demographic details, smoking history, duration of presenting symptoms, time from presentation to diagnosis, diagnostic investigations, and initial treatment plan. Clinicians were asked to record the usefulness of each diagnostic test with respect to diagnosis and further management and their overall treatment intent. The completeness of the registration database was checked against histopathological registers serving the area.

At the end of the registration period (29 February 1992) a follow up form was completed to document the actual treatment received by patients and to register initial follow up. An interval of at least six months from registration had elapsed in all patients. Information was obtained from the consultant responsible for the initial diagnosis, case note extraction in the regional cardiothoracic and oncology centres for patients who underwent surgery, radiotherapy and chemotherapy, and by postal enquiry to general practitioners in patients receiving supportive care in the community. Information was sought from case notes on treatment actually received, response to treatment and complications using standard evaluation criteria,${ }^{2}$ length of inpatient stay in all cases and, in all except the surgical patients, symptom palliation. Date of death or date when last seen was recorded for all patients. For those patients still alive at the time of the completion of the follow up form a further enquiry to their general practitioner was conducted in April/May 1993 which established the survival status of the entire population.
Received 18 November 1994 6 April 1995

Revised version received 29 February 1996 Accepted for publication 5 March 1996 


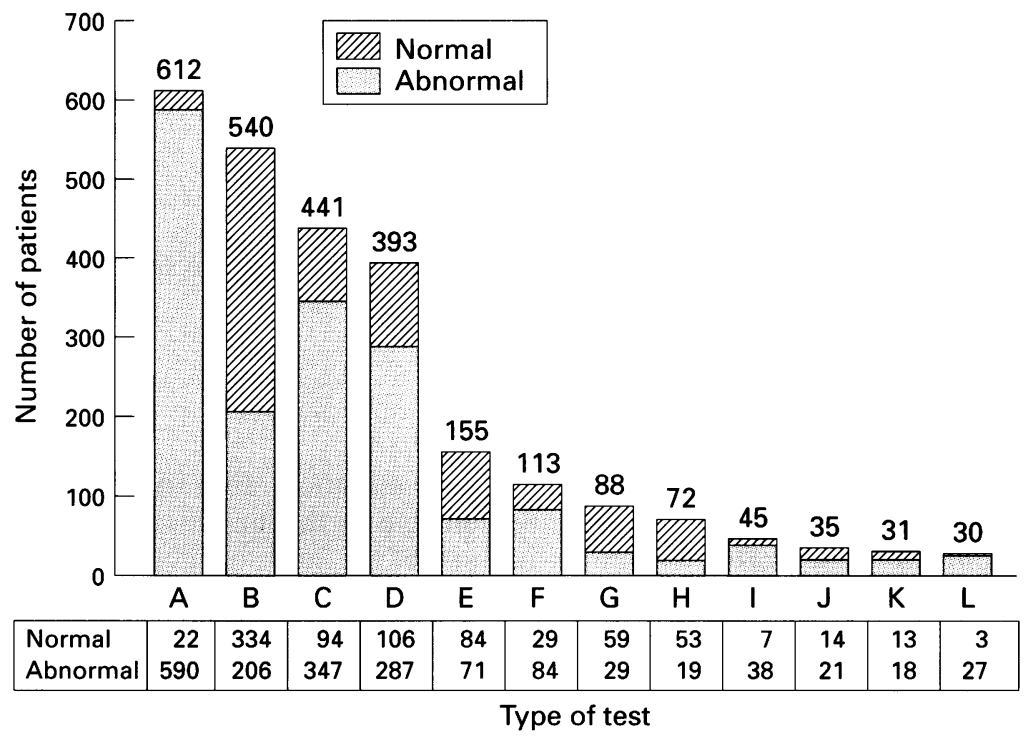

Figure 1 Investigations performed at the time of diagnosis $(n=622) . A=$ chest radiography; $B=$ blood tests; $C=$ bronchoscopy; $D=$ bronchial biopsy/brushing; $E=$ sputum cytology; $F=C T$ scan of thorax; $G=$ ultrasound abdomen; $H=$ barium swallow; $I=$ extrathoracic fine needle aspiration; $\mathcal{F}=$ pleural aspiration; $K=$ pleural biopsy; $L=$ thoracic fine needle aspiration. The figures at the top of each column represent the number of patients tested.

Table 1 Treatment proposed compared with treatment delivered

\begin{tabular}{lccccc}
\hline $\begin{array}{l}\text { Treatment } \\
\text { proposed }\end{array}$ & \multicolumn{2}{l}{ Treatment delivered } & Total \\
\cline { 2 - 5 } & Surgery & Radiotherapy & Chemotherapy & Supportive care & \\
\hline Surgery & 128 & 6 & 2 & 8 & 144 \\
Radiotherapy & 1 & 142 & 1 & 24 & 168 \\
Chemotherapy & 1 & 12 & 75 & 7 & 94 \\
Supportive care & 1 & 8 & 1 & 170 & 180 \\
Total & 130 & 168 & 79 & 209 & 586 \\
\hline
\end{tabular}

Table 2 Patient characteristics in main treatment groups

\begin{tabular}{lccl}
\hline & $\begin{array}{c}\text { Curative } \\
(n=151)\end{array}$ & $\begin{array}{c}\text { Palliative } \\
(n=471)\end{array}$ & $p$ value \\
\hline Age (mean) & $64 \cdot 9$ & $68 \cdot 8$ & $<0 \cdot 001$ \\
M:F & $103: 48$ & $316: 155$ & NS \\
Weight loss & 37 & 185 & $<0 \cdot 001$ \\
$\quad$ Yes & 114 & 286 & $<0 \cdot 001$ \\
$\quad$ No & $139(92 \%)$ & $212(45 \%)$ & $<0 \cdot 001$ \\
Performance status 0/1 & $4 \cdot 9$ & $4 \cdot 2$ & NS \\
Mean no. of tests performed & $59 \cdot 2$ & $73 \cdot 3$ & $<0 \cdot 01$ \\
Mean length of history (days) & $132(97 \cdot 4 \%)$ & $378(80 \cdot 3 \%)$ & \\
Positive histology & 44 & 49 & $\mathrm{NS}$ \\
Length of history & $0-294$ & $0-1095$ & \\
$\quad$ Median & & & \\
Range & & & \\
\hline
\end{tabular}

\section{Results}

In the study period the group registered 622 patients (419 men) with lung cancer. Follow up forms documenting subsequent management were obtained for $586(94 \%)$. The mean age was 68 years (range $36-90$ ); $80 \%$ of patients were referred to respiratory physicians directly from primary care and the other $20 \%$ were referred by other hospital consultants. Four hundred and sixteen patients $(67 \%)$ were current smokers, $180(29 \%)$ were ex-smokers, and only 26 patients $(4 \%)$ had no history of cigarette consumption. Two hundred and twenty two patients $(35 \cdot 7 \%)$ had evidence of significant weight loss ( $>10 \%$ body weight) in the previous six months. ECOG performance status ${ }^{2}$ at pre- sentation was 0 in $18.6 \%, 1$ in $37.8 \%, 2$ in $26.2 \%, 3$ in $13.7 \%$, and 4 in $3.7 \%$. The time from the first tumour-related symptom to referral to hospital (length of history) showed a considerable variation with a median duration of 45 days, range (0-1095). Only 39\% of patients were referred within a month from first experiencing significant symptoms. A clinical diagnosis was made on the day of referral in $52 \%$ and confirmed in $87 \%$ of patients within two weeks. Inpatient assessment at presentation (mean duration of 5.8 days) was performed in $403(65 \%)$ cases. Histological or cytological confirmation was obtained in 510 cases $(82 \%)$, of which $38 \%$ were squamous, $24.5 \%$ small cell, $16 \%$ adenocarcinomas, $5 \%$ large cell, and $16.5 \%$ undifferentiated. The investigations performed at the time of diagnosis and the percentage of abnormal results are summarised in fig 1 . Chest radiography was abnormal in most of the patients $(97 \%)$ and contributed to diagnosis in $90 \%$. Bronchoscopy was performed frequently $(71 \%)$ and was highly informative (78.4\% abnormal) and diagnostic in $73 \%$ of patients. It was the main source of histological confirmation. Sputum cytological examination was performed in a quarter of patients and was positive in almost half of those studied (46\%). Fine needle aspirates from thoracic and extrathoracic sites gave a high level of diagnostic positivity (thoracic $27 / 30(90 \%)$, extrathoracic $38 / 45(84 \%)$ abnormal).

Blood tests (haematological and biochemical screening) were performed on $87 \%$ of patients but were abnormal in only $38 \%$ and contributed to diagnosis in only $10 \%$ or to management in $32 \%$. More sophisticated radiological investigations were performed infrequently. Computed tomographic (CT) scanning, which was performed in $18 \%$ of patients, was highly informative $(74.3 \%$ abnormal $)$ compared with a barium swallow (26\% abnormal in the $12 \%$ of patients studied). A highly significant association ( $p<0.001, \chi^{2}$ test) was found between initial referring hospital and number of investigations performed $(\leqslant 3$ and $\geqslant 6$ tests/patient). The proposed management following diagnosis and initial evaluation was surgery in $144(24.5 \%)$ cases, radiotherapy in $168(29.5 \%)$, chemotherapy in $94(16 \%)$, and supportive care in $180(30 \%)$. The relationship between proposed and delivered treatment can be seen in table 1 . For the majority $(75 \cdot 7 \%)$, treatment intent was palliative. Of the patients who were considered suitable for curative treatment ( $\mathrm{n}=151$ ), 135 were referred for surgery. As a group these patients were younger, had better performance status, less often had weight loss, and had a shorter history and inpatient stay during their diagnostic evaluation (table 2 ). Their overall survival was much better than those treated with palliative intent (fig 2). Overall survival was poor (fig 3 ).

\section{SURGERY}

Preoperative CT scans were obtained from 47 of the 130 resected patients $(36 \cdot 2 \%)$. In 71 patients $(54 \cdot 6 \%)$ mediastinal nodes were 
Table 3 Postoperative pathological staging ( $p T N)$ of surgically resected patients

\begin{tabular}{lrrrlr}
\hline & $N 0$ & $N 1$ & $N 2$ & $N X$ & Total \\
\hline T1 & 12 & 8 & 1 & 0 & 21 \\
T2 & 31 & 26 & 18 & 4 & 79 \\
T3 & 7 & 11 & 4 & 0 & 22 \\
T4 & 0 & 0 & 3 & 1 & 4 \\
TX & 0 & 1 & 0 & 3 & 4 \\
Total & 50 & 46 & 26 & 8 & 130 \\
\hline
\end{tabular}

sampled either at mediastinoscopy $(n=39)$ or at operation $(n=32)$, although a frozen section facility was not routinely available. Fifty five patients underwent pneumonectomy, 68 lobectomy, and six segmental or thoracoscopic resections. The median inpatient stay was 12 days (range 3-101); six patients died within 30 days of operation (5\%).

Pathological staging (pTN) is summarised in table 3. Twenty six patients had pathological evidence of $\mathrm{N} 2$ disease after operation. This is likely to be an underestimate in view of the fact that $45 \%$ of operated patients did not undergo mediastinal node sampling. Of the 130 surgical patients $62(48 \%)$ had squamous histology, $23(18 \%)$ had adenocarcinomas and large cell cancers, and six ( $4 \cdot 6 \%)$ had small cell subtypes. Twenty four patients $(19 \cdot 8 \%)$ were referred for postoperative radiotherapy and six $(4 \cdot 6 \%)$ had subsequent chemotherapy. One patient had two operative procedures and 97 patients $(74 \cdot 8 \%)$ were followed up but not offered further treatment until relapse. Survival of surgically treated patients was highly dependent on $\mathrm{T}$ or $\mathrm{N}$ status (fig $4 \mathrm{~A}$ and $4 \mathrm{~B}$ ).

\section{RADIOTHERAPY}

One hundred and sixty eight patients (27\%) received radiotherapy as their first treatment. Treatment intent was palliative in 129,10 patients were treated with radical radiotherapy and curative intent, 26 received postoperative "adjuvant" radiotherapy, and in 3 the treatment intent was not specified. Twenty patients $(11 \cdot 8 \%)$ were treated as part of a research protocol. The radiotherapy treatment was to the primary site in 118 patients $(71.4 \%)$, to bone metastases in $12(7 \cdot 1 \%)$, and to brain metastases in $10(5.9 \%)$. In the remaining $16 \%$

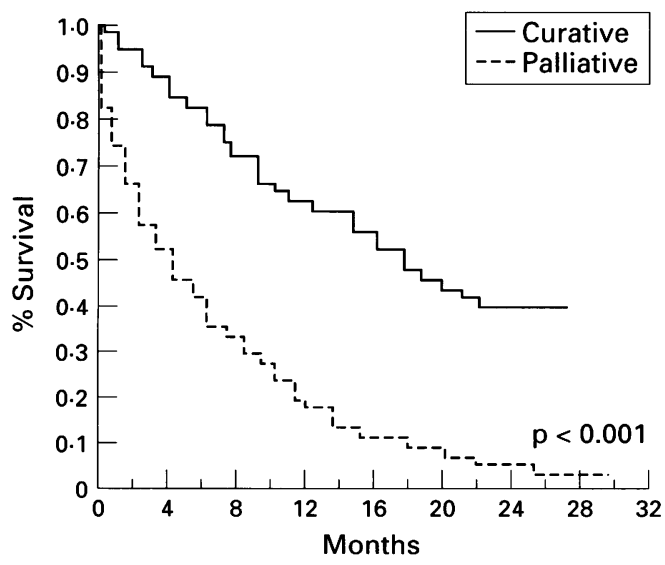

Figure 2 Overall survival for patients treated with palliative/curative intent.

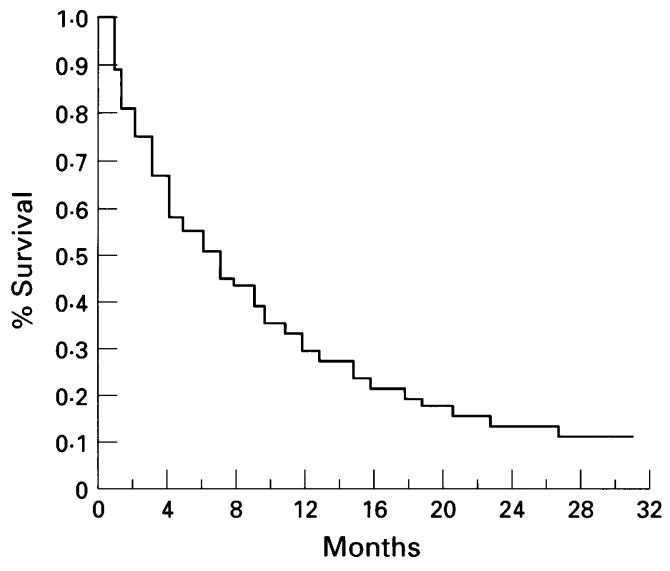

Figure 3 Survival for all patients from the date of diagnosis

of patients other sites of metastases were irradiated (skin, lymph nodes, etc). Twenty seven patients $(16 \%)$ received a single fraction, 52 (31\%) five fractions, $54(32 \%) 10$ fractions, and for 10 patients $(6 \%)$ the treatment was radical with 20 fractions. In 25 patients there was no record in the case notes of the number of fractions given. Radiation doses varied from $8 \mathrm{~Gy}$ in a single fraction to $52.5 \mathrm{~Gy}$ for a radical course of treatment. Twenty seven patients received a total dose of less than $20 \mathrm{~Gy}$. Eighty seven patients $(52 \%)$ were treated as outpatients; $98 \cdot 2 \%$ of patients received their treat-
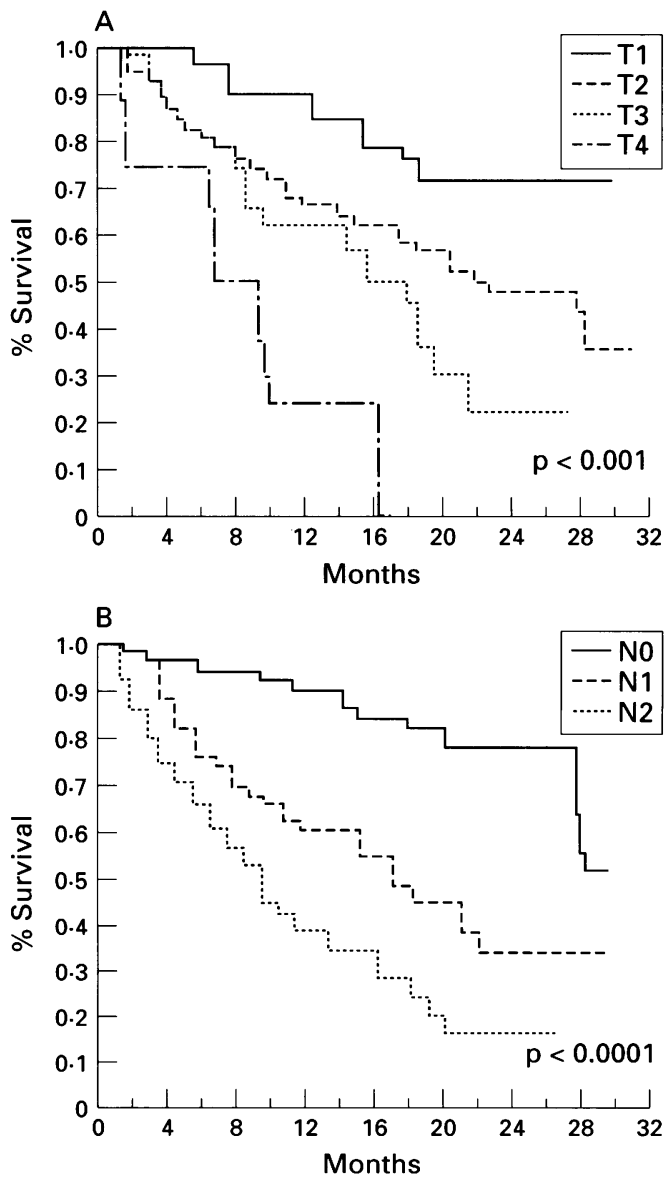

Figure 4 Survival of surgically resected patients according to (A) $T$ stage and (B) $N$ stage. 


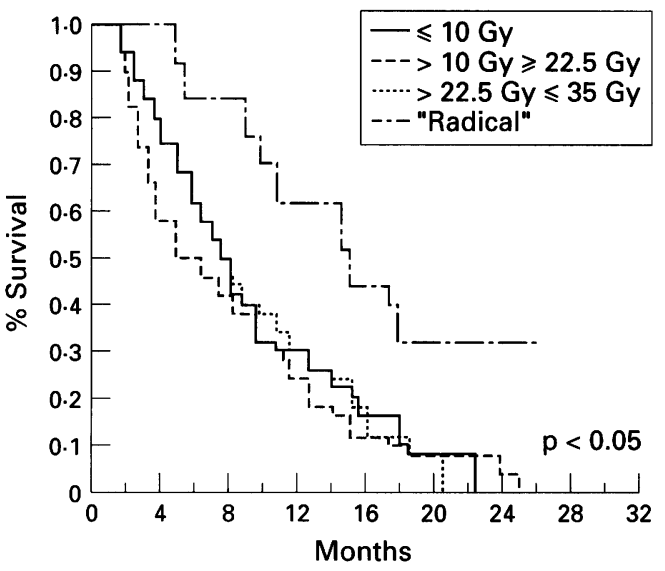

Figure 5 Survival according to radiation dose. No difference in survival rates was seen between the three palliative regimens, but significant $(p=0.02)$ survival was seen in the radical group compared with palliative treatment.

ment in less than four weeks and $69 \cdot 8 \%$ in less than seven days of inpatient treatment.

Objective responses and symptom improvement were assessed at four weeks in 54 $(32 \%)$ and $124(74 \%)$ patients, respectively. Of these there was radiological improvement in $26(48 \%)$ and improvement in symptoms in $87(70 \%)$. Complications of radiation were noted in $46(27 \%)$ of treated patients; 122 patients $(73 \%)$ had none.

Survival was poor with an overall median survival of seven months; $147(87 \cdot 5 \%)$ patients were dead one year after treatment. The influence of radiation dose on survival can be seen in fig 5 which shows little difference in survival between dose levels up to $30 \mathrm{~Gy}$, but a significant difference between these and radical doses of irradiation. The small group of patients treated with curative intent had a median survival of 14 months, one year survival of $60 \%$, and two year survival of $30 \%$.

\section{CHEMOTHERAPY}

Seventy nine patients received chemotherapy. Seven main types of treatment regimen could be identified and $60(75.9 \%)$ patients were treated outside a research protocol. The mean number of courses given was 3.5. Sixteen patients $(20.3 \%)$ received six courses and 12 patients $(15 \%)$ only one. Complete remission was seen in $16(20 \%)$ and a partial response in $26(33 \%)$; symptoms were abolished in 22 patients $(28 \%)$ and improved in a further 24 $(30 \%)$. Side effects were unspecified in 34 patients, alopecia developed in 20 , nausea and vomiting in 18, and haematological toxicity in six. Sixty one patients had small cell histology. The mean total inpatient stay was $13 \cdot 1$ days with a median of 12 days (range 1-68). Sixteen $(20 \%)$ of patients were treated as outpatients or day cases. Survival results are illustrated in fig 6 . The median survival for all patients receiving chemotherapy was 238 days. Patients with small cell histology $(n=61)$ had a median survival of 225 days and those with non-small cell tumours $(n=18) 269$ days. No discernible

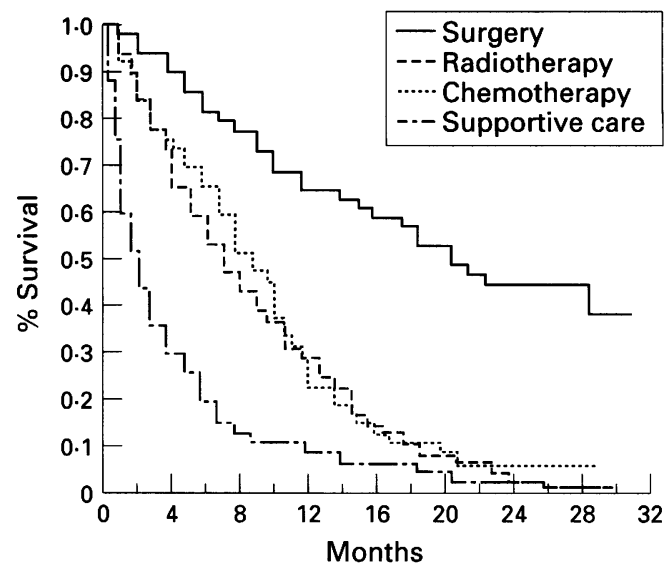

Figure 6 Influence of treatment on survival.

survival difference could be found between the chemotherapy regimens used.

\section{SUPPORTIVE CARE}

Of the 209 patients in this category where a follow up form was completed $(n=166)$, care was delivered by the patient's general practitioner in 153, four had initially been referred to a hospice, and nine were followed up by the hospital which made the initial diagnosis. In the 132 patients $(63.2 \%)$ who were assessed within the first year, symptoms were abolished in three, improved in $68(32.5 \%)$, unchanged in 45 , and worse in 16 . In 77 patients $(36.8 \%)$ the impact of supportive care on symptoms was not known. Subsequent inpatient hospital stay was required in 124 patients with a median duration of 10 days (range 1-87). Survival was poor (median 2 months) with only $10 \%$ of patients surviving one year (fig 6).

\section{Discussion}

Reports describing management practice and outcomes in unselected groups of patients with lung cancer are rare. ${ }^{34}$ The South East of Scotland Lung Cancer Group has a tradition of registration audits ${ }^{1}$ and set out to document prospectively present diagnostic and management practices. The aim was not to register all patients with lung cancer in the area of Lothian, Fife and Borders, but those whose initial assessment and further active treatment was undertaken by the SESLCG members. This encompasses all patients managed by respiratory physicians and thoracic surgeons in this area. Audit returns were compared with registers of pathological diagnoses of all subtypes of lung cancer for this period in the two main and one subsidiary pathology departments serving the area. In addition, the number of patients with ICD9 diagnosis 162 (lung cancer) for 1991 and 1992 in all the geographical areas covered by our group were obtained from the Scottish Cancer Registry. ${ }^{5}$ By comparing these two data sets we established that we have registered $62 \%(662 /$ $1073)$ of the total and $63 \%(317 / 506)$ of the patients less than 70 years of age. The geographical area served by the Cancer Registry 
does not correspond to that covered by SESLCG. Many patients from North East Fife district are referred to Dundee and some patients in West Lothian are referred to Falkirk or Stirling which receives regional services from Glasgow. The proportion of patients on whom ICD9 162 diagnosis is made without histological confirmation and without referral to a respiratory physician or thoracic surgeon is also difficult to estimate accurately. The subset of patients managed by the members of SESLCG, who have a special interest in lung cancer, includes most of the patients receiving active treatment and a high level of complete follow up $(94 \%)$ was achieved in this audit.

This study confirms previously reported epidemiological data of unselected groups with lung cancer. ${ }^{134}$ It is a disease which affects predominantly older men who are current or ex-cigarette smokers. Whilst screening of high risk groups has not been found to be effective, ${ }^{6}$ repeated studies of outcome in asymptomatic patients detected as prevalence cases have shown a higher frequency of early stage tumours and better prognosis with appropriate treatment. ${ }^{7}$ Most patients in the study were symptomatic at presentation, but the length of history (average 2.5 months before hospital referral) suggests the difficulty of identifying significant new symptoms in this population. Early diagnosis is therefore important and prompt identification of patients requiring specialist assessment necessary. This may need education and guidelines for providers of primary care and more ready access to specialist respiratory assessment. Once referred, diagnosis was usually prompt and histologically confirmed in most patients $(82 \%)$. This reflects the widespread use of fibreoptic bronchoscopy and availability of this diagnostic procedure often on an outpatient basis.

Various other investigations were performed as part of the initial assessment. The selection of investigations was influenced more by geographical location and the practice of individual clinicians than the proposed therapeutic aim. For example, the proportion of patients in the "curative" treatment category undergoing thoracic CT scans varied from $6.6 \%$ to $91 \%$ in different hospitals. In the group of 440 patients referred for "palliative" treatment the differences in frequency of CT scanning were less marked $(7-43 \%)$. Just under one quarter of patients were judged suitable for "curative" treatment and the majority of these (146/151) were referred for surgery. Preoperative evaluation of mediastinal nodal involvement was not routine. Preoperative CT scanning, performed in only $38 \%$ of all these patients, was often not done due to the lack of availability and perhaps to the substantial waiting time at certain centres for this investigation. Considerable geographical variation in the frequency of preoperative CT scanning was observed. The highest rates came from the district general hospital with a recently installed CT scanner (91\%) and the lowest (10\%) from a geographically remote area which did not have a local scanner. Mediastinoscopy was also generally underused $(32 \%)$, and these two factors may account for the low documented incidence of $\mathrm{N} 2$ disease $(20 \%)$ in the surgical population. A recent postal survey ${ }^{8}$ has shown that the perioperative staging practices used by the SESLCG are similar to the rest of the UK. Duration of inpatient stay and postoperative mortality are comparable with other published series. $^{34}$

In order to select patients suitable for surgical resection we need accurate preoperative staging. Evaluation of mediastinal nodal status will be particularly relevant if the promising results of additional chemotherapy and radiation for this group of patients are confirmed. ${ }^{9}$ Separation of the minority of patients with purely localised disease (N0) from the rest has major prognostic importance. Attempts to increase the proportional size of this group should include early referral and prompt and accurate diagnosis. This will need education of both primary care physicians and high risk patient groups.

The outcome for the patients who received radiotherapy confirms the advanced and often metastatic nature of lung cancer at presentation. The small number of patients suitable for primary chest irradiation who were selected for high dose treatment with "curative" intent achieved a two year survival of $30 \%$ with twice the median survival of the patients treated palliatively. That long term survival comparable to surgical results can be achieved immediately in operable patients with high dose irradiation has been demonstrated in previous studies. ${ }^{10}$ However, as only 10 patients were treated in this way, the impact on overall survival for all patients treated with radiotherapy is probably small. For most patients, however, radiation dose or schedule had little influence on the outcome. This finding confirms results of previous studies ${ }^{11} 12$ and suggests that treatmentrelated prognostic factors are outweighed by the natural history of lung cancer. As would be appropriate for this palliative situation, objective tumour responses to radiotherapy were frequently not documented; however, $70 \%$ of symptomatic patients had fewer symptoms following irradiation. Details of degree of improvement and duration of symptom control are not available in this study.

Chemotherapy has been used rarely $(13.5 \%$ patients), reflecting the general perception of low effectiveness and potential toxicity. ${ }^{13}$ Even amongst the most chemosensitive histological variety (small cell lung cancer) less than half $(61 / 126)$ of patients received systemic treatment in spite of the fact that this group of patients was younger $(60 \%<70$ years $)$ than the average. Many different drug regimens were used without discernible impact on survival. Response rates and survival figures were slightly poorer than expected. ${ }^{14}$ The better survival for the small subgroup of patients with non-small cell histology was surprising and likely to result from selection bias.

Not surprisingly, it was difficult to obtain detailed follow up information on patients who received supportive care. Most were managed in the community but many required subsequent inpatient management. Their survival 
was poor with a median of two months. As a group, few appeared to have an initial hospice referral, although we have no data on subsequent involvement of the palliative care services.

The Standing Medical Advisory Comittee ${ }^{14}$ has recently attempted to characterise current clinical practices but, as yet, no generally accepted national or international guidelines for the management of lung cancer are available. This is despite the fact that it is the most common malignant disease in the western world and many different treatment strategies are in current clinical use. A recent prospective population-based study ${ }^{15}$ in another common tumour, breast cancer, has shown a marked lack of consensus in management amongst clinicians. This study provides an insight into the current investigation and management practices in an unselected group of patients with lung cancer and may be useful as a baseline against which to test proposed guidelines for the management of this common malignancy.

We are grateful to all our colleagues in the South East of Scotland Lung Cancer Group: Professor C Haslett, Dr NJ Douglas, Dr W MacNee, Dr M Sudlow, Dr AG Leitch, Dr GK Crompton, Dr AP Greening, Dr GJR McHardy, Mr EW GK Crompton, Dr AP Greening, Dr GJR McHardy, Mr EW Choo Kang, Dr GR Petrie, Dr D Lamb, Dr MA MacIntyre, Professor JF Smyth, Dr GA Newaishy, Dr RCF Leonard; $\mathrm{Mr}$ Stuart Griffen for his assistance with data collection, and Mrs Fiona Penman for typing the manuscript. The study was supported by the Lothian Area Audit Committee. Dr Anna Gregor is supported by the Imperial Cancer Research Fund.
1 Edinburgh Lung Cancer Group. Patients presenting with lung cancer in South East Scotland. Thorax 1987;42. lung canc

2 WHO. Handbook for reporting results of cancer treatment. WHO offset publication No. 48. Geneva: WHO, 1979.

3 Connolly CK, Jones WG, Thorogood J, Head C, Muers MF. Investigation, treatment and prognosis of bronchia carcinoma in the Yorkshire region of England. Br $\mathcal{F}$ Cancer 1990;61:579-83.

4 Watkin SW, Hayhurst GK, Green JA. Time trends in the outcome of lung cancer management: a population study of 9090 cases. Br f Cancer 1990;61:590-6.

5 Scottish Cancer Registry, Information and Statistics Division, Directorate of Information Services NHSiS, January 1994 .

6 Huhti E, Saloheimo M, Sutinen S. The value of roentgenologic screening in lung cancer. Am Rev Respir Dis 1983;128:395-8.

7 Fontana RS. Screening for lung cancer: recent experience in the United States. In: Hansen $\mathrm{HH}$, ed. Lung cancer: basic and clinical aspects. Boston: Martinus Nijhoff, 1986

8 Tsang GMK, Watson DCT. The practice of cardiothoracic surgeons in the perioperative staging of non-small lung cancer. Thorax 1992;47:3-5.

9 Shepherd FA. Induction chemotherapy for locally advanced non small cell lung cancer. Ann Thorac Surg 1993;55: 1585-92.

10 Burt PA, Hancock BM, Stout R. Radical radiotherapy for carcinoma of the bronchus: an equal alternative to radical carcinoma of the bronchus: an equal

11 Medical Research Council Lung Cancer Working Party. Inoperable non-small-cell lung cancer: Medical Research council randomised trial of palliative radiotherapy with two fractions or ten fractions. BrF Cancer 1991;63:265-70

12 Bleehen NM, Girling DJ, Machin D, Stephens RJ on behal of the MRC Lung Cancer Working Party. A Medical Research Council (MRC) randomised trial of palliative radiotheraphy with two fractions or a single fraction in patients with inoperable non-small cell lung cancer (NSCLC) and poor performance status. Brf Cancer 1992; 65:934-41.

$13 \mathrm{McVie}$ JG. Chemotherapy of non-small cell lung cancer Ann Oncol 1993;4:804-5.

14 Standing Medical Advisory Committee. Management of lung cancer: current clinical practices. Report of a working group chaired by Professor JMA Whitehouse, 1994

15 Chouillet AM, Bell CMJ, Hiscox JG. Management of breas cancer in Southeast England. BMF 1994;308:168-71. 